\title{
Hegels Grundlinien der Philosophie des Rechts und Spinozas Politischer Traktat- Parallelen in der Architektonik mancher Begriffe
}

\section{Hegel's Philosophy of Right and Spinoza's Political Treatise- Parallelism in the Construction of some Concepts}

\begin{abstract}
ABSTRAKT: Hegel und Spinozas Theorien haben ganz verschiedene Ausgangspunkte. Doch bei einer genaueren Analyse kann man bei vielen Begriffen und Gedankengängen Parallelen in der Architektonik finden. Es werden Begriffe wie, vernünftiger und beständiger Wille von Spinoza und der objektive Wille bei Hegel, die Beziehung zwischen dem Naturzustand und Gesellschaftlichen Zustand in Bezug auf den Begriff Natur, die List der Vernunft in der Institutions-Logik und auch die Bedeutung von dem Macht-Begriff in den zwischenstaatlichen Beziehungen analysiert.
\end{abstract}

SCHLÜSSELBEGRIFFE: HEGEL - SPINOZA - VERNÜNFTIGER WILLE - NATURZUSTAND - GESELLSCHAFTLICHER ZUSTAND - INSTITUTIONEN - MACHT ZWISCHENSTAATLICHE BEZIEHUNGEN.
ABSTRACT: Hegel and Spinoza's theories have different points of departure, but with a precise analysis we can see, that in the construction and train of thought, that they have parallel views in many concepts. We will analyse concepts like rational will, objective will, nature, natural state and civil state, the institutions-logic and the power concept in the international law in this context.

KEY WORDS: HEGEL - SPINOZA - OBJECTIVE WILL - NATURAL STATE CIVIL STATE - INSTITUTIONS - POWER - INTERNATIONAL LAW 
$\mathrm{H}^{\mathrm{s}}$ egels Grundlinien der Philosophie des Rechts und Spinozas Politischer Traktat sind zwei klassische Werke der politischen Philosophie. Bei dem Aufbau dieser beiden Werke kann man auf manche Parallelen stoßen, ins besondere bei manchen Begriffen. Doch ist es nicht ganz so einfach die Parallelen herauszuarbeiten, weil Hegel in den Grundlinien der Philosophie des Rechts Spinoza fast gar nicht erwähnt. Doch trotzdem ist Spinoza in diesem Werk präsent. Über Spinozas Einfluss in den Grundlinien gibt es viele Werke. Doch über den Einfluss des Tractatus Politicus, den Hegel in seinem Werk nicht zitiert, kann man nicht sehr viel finden. Explizit zitiert Hegel Spinoza in den Grundlinien nur einmal in Bezug auf den causa sui Begriff. Wir wissen, dass Spinozas Gesamte Werke Hegel schon früh bekannt sind. ${ }^{1}$ Aus seinen Schriften, wissen wir, dass er die Ethik, Descartes' Prinzipien der Philosophie, die Briefe ${ }^{2}$ und den Tractatus Theologico-Politicus gelesen hat. In den Vorlesungen über die Geschichte der Philosophie bezeichnet er die Ethik als Hauptwerk, während er den Tractatus Theologico-Politicus nur am Rande erwähnt. Was den Tractatus Politicus (TP) angeht, so verliert Hegel kein Wort darüber, obwohl dieser im selben Band der Paulus-Ausgabe, an der auch Hegel mitgearbeitet hat, zu Hegels Zeiten auch schon als Übersetzung von Ehwald ${ }^{3}$ vorhanden war. Auch wenn wir außer einem kurzen Zitat aus der Ethik nichts Explizites in den Grundlinien finden können, können wir viele Parallelen durch eine Analyse mancher Begriffe ziehen.

\section{Der beständige Wille bei Spinoza UND DER OBJEKTive Wille bei Hegel}

Bei Hegel basieren die Grundlinien der Philosophie des Rechts auf den Begriff des «freien Willens» ${ }^{4}$, der auf den ersten Blick einen Kontrast zu dem

[1] Hegel hat an der Spinoza Edition von Paulus mitgearbeitet und die Gallica Edition bezüglich der Fußnoten verglichen (LuCAs, H 1982-83, «Hegel et l'édition de Spinoza par Paulus (1)», Cahiers Spinoza, Numéro 4, Editions Réplique, p. 136).

[2] Von den Briefen erwähnt Hegel in der Geschichte der Philosophie den 36. Brief über das Böse (Hegel, GWF 2003, Vorlesungen über die Geschichte der Philosophie III, Suhrkamp Verlag, Frankfurt am Main, p. 192).

[3] Lucas vermutet, dass Hegel den Politischen Traktat zwar nicht in der Steigerschen Bibliothek nachschlagen konnte, wohl aber in der Bibliothek Hufnagels in Frankfurt (LuCAs, H 1999, 'Der junge Hegel zwischen Revolution und Reform. Politische und rechtsphilosophische Optionen Hegels Übergang von Bern nach Frankfurt', Hegels Denkentwicklung in der Berner und Frankfurter Zeit, Ed. M. Bondeli; H. Linneweber-Lammerskitten, Fink Verlag, München, p. 274).

[4] In den Grundlinien der Philosophie des Rechts heißt es: «Der Boden des Rechts ist überhaupt das Geistige und seine nähere Stelle und der Ausgangspunkt der Wille, welcher frei ist.» (R § 4) (HegeL, GWF 1986, Grundlinien der Philosophie des Rechts, Suhrkamp Verlag, 
Selbsterhaltungstrieb Spinozas darstellt. Aber bei einer genaueren Analyse sieht man, dass Hegels freier Wille einen ähnlichen Prozess wie Spinozas beständiger oder vernünftiger Wille durchläuft. Der Wille ${ }^{5}$, der bei Spinoza und bei Hegel als vernünftiger Wille ausgedrückt wird, hat die gleiche Perspektive, nämlich, dass dieser beständige Wille nur durch Einsicht erreicht werden kann. Die Problematik wird bei Spinoza im zweiten Teil des Politischen Traktats und bei Hegel am Anfang der Grundlinien behandelt. In den Grundlinien der Philosophie des Rechts und auch in dem Politischen Traktat, können wir einen Werdegang von dem unmittelbaren Trieb zum vernünftig bestimmten Willen finden. Auch wenn bei Hegel die Problematik doch mehr in der Phänomenologie des Geistes gelagert ist, so finden wir auch in den Grundlinien einen Prozess vom unmittelbaren Trieb zum vernünftig bestimmten Willen. ${ }^{6}$

Die Entwicklung vom unmittelbaren Trieb zum vernünftig bestimmten Willen ist bei Spinoza und auch bei $\mathrm{Hegel}^{7}$ ein innerlicher Prozess, der aber gleichzeitig äußerliche Folgen hat. Die Dialektik der Anerkennung kann mit dem Zustand esse sui iuris und esse alterius iuris bei Spinoza verglichen werden. ${ }^{8}$ Der Gestaltwandel vom unmittelbaren Trieb zum vernünftigen Willen ist zugleich die Entwicklung von Fremdbestimmtheit (esse alterius iuris) zur Selbstbestimmtheit (esse sui iuris). Die Begriffe ,Selbstbestimmtheit' und ,Fremdbestimmtheit' bei Spinoza ${ }^{9}$ und die Herr-Knecht-Beziehung Hegels können in zwei Dimensionen - nämlich in den geistigen und der körperlichen - analysiert werden. Dabei wird die Beziehung zwischen geistiger und körperlicher Knechtschaft deutlich. Der Übergang von der Fremdbestimmtheit zur Selbstbestimmtheit bei Spinoza und auch der Prozess der Anerkennung bei Hegel ist ein Bewusstseinsprozess, der mit dem Denken und den adäquaten

Frankfurt am Main, p. 46.)

[5] Im Politischen Traktat beschreibt, Spinoza den beständigen Willen: «Gehorsam (...) ist der beständige Wille, dasjenige auszuführen, was dem Recht nach gut ist und was kraft eines gemeinsamen Beschlusses geschehen soll» (SpINozA, B 1994, Politischer Traktat, Felix-Meiner Verlag, Hamburg, p. 31.)

[6] Hegel benutzt den Begriff «freier oder allgemeiner Wille» und Spinoza «fester oder beständiger Wille» (voluntas constans), um aber die Ähnlichkeit herausarbeiten zu können habe ich den Begriff vernünftig bestimmter Wille benutzt, weil beide Denker diesen Begriff durch die «Vernunft» zu erklären versuchen.

[7] Hegel nach besteht der unmittelbare oder natürliche Wille von Trieben Begierden und Neigungen (Hegel, GWF 1986, Grundlinien der Philosophie des Rechts, Suhrkamp Verlag, Frankfurt am Main, p. 62).

[8] Macherey, A 1992, Avec Spinoza, Etudes sur la doctrine de l'histoire du spinozisme, PUF, Paris, p. 137.

[9] Diese Begriffe werden im zweiten Teil des Politischen Traktats entwickelt. 
Ideen eng verbunden ist. Durch die Übereinstimmung von Denken und Wollen kann sich bei Spinoza und Hegel auch ein vernünftiger Wille entwickeln. ${ }^{10}$

In der Beziehung zwischen den Affekten und der Vernunft besteht eine Grundproblematik, welche die Voraussetzung für das Verständnis des Aufbaus der Werke beider Denker ist. Der Gedanke, dass die Menschen zwischen Leidenschaft und Vernunft schwanken und durch die Mitmenschen mehr Möglichkeiten, sich zu verwirklichen und sich einen freien Handlungsraum zu erschaffen haben ist zu sehen. Das Ziel des Staates ist sowohl bei Spinoza als auch bei Hegel die Realisierung der Freiheit. ${ }^{11}$ Der Einfluss Spinozas auf Hegel ist in seinen Begriffen der «Macht» und der «Natur» sichtbar und beeinflusst auch dessen Auffassung von «Freiheit». Es ist ein wichtiger Punkt, den Hegel übernommen hat, die Freiheit nicht als Gegensatz zur Notwendigkeit zu verstehen und zu interpretieren. Das beeinflusst auch seine ganze Staatstheorie. Freiheit besteht nicht darin, das zu tun, was man gerade will. Freiheit entsteht in einem Bildungsprozess und benötigt ein hohes Bewusstseinsvermögen.

Die Tatsache, dass das Natürliche, das Triebhafte im Menschen niemals ganz verschwindet, nur durch Bildung in der Gesellschaft kultiviert werden kann, sehen beide Denker. Dem Übergang von der imaginatio zur ratio legt bei Hegel ähnliche Motive wie Spinoza zugrunde. Auch bei ihm ist es ein innerlicher Prozess, durch den der Mensch sich von der Knechtschaft der Leidenschaften befreit. Trieb und Wille sind sowohl bei Spinoza als auch bei Hegel ${ }^{12}$ nicht getrennt, sondern sind miteinander verknüpft. Das hängt mit dem Naturrechtsbegriff beider Denker eng zusammen. Bei Spinoza hört das Naturrecht des Menschen im gesellschaftlichen Zustand nicht auf. Diese triebhafte Natur kann sich durch das Denken von den inadäquaten Ideen, die Leiden verursachen, in adäquate Ideen, also in Aktivität umwandeln, und gerade darin sieht Spinoza das Vernünftige. Aber auch wenn der Mensch in manchen Situationen die Einsicht hat und vernünftig handelt, heißt das nicht, dass er immer so handeln wird. Das Schwanken zwischen der Unvernunft wegen inadäquater Ideen und

[10] Walther, M 1991, 'Gemeinschaft und Gesellschaft bei Ferdinand Tönnies. Philosophisch - anthropologische Grundlagen einer künftigen Analyse', Hundert Jahre "Gemeinschaft und Gesellschaft», Ferdinand Tönnies in der internationalen Diskussion, Ed. L Clausen, C Schlüter, Leske und Budrich, Opladen, p. 91.

Rотн, K 1991, Die Institutionalisierung der Freiheit in den Jenaer Schriften Hegels, Schäuble Verlag, Rheinfelden, p.248.

[11] Nach Hegel ist nur der Wille «als denkende Intelligenz» freier Wille. (R \$21) (HeGEL, GWF 1986, Grundlinien der Philosophie des Rechts, Suhrkamp Verlag, Frankfurt am Main, p. 72.)

[12] Hegel erkennt in den Grundlinien der Philosophie des Rechts, «dass die Triebe als das vernünftige System der Willensbestimmung seien; sie aus dem Begriffe zu fassen, ist der Inhalt der Wissenschaft des Rechts» (Hegel, GWF 1986, Grundlinien der Philosophie des Rechts, Suhrkamp Verlag, Frankfurt am Main, p. 70). 
der Vernunft, die von adäquaten Ideen bestimmt wird, ist immer vorhanden. Der Übergang vom natürlichen Zustand zum gesellschaftlichen ist also kein fiktiver, sondern ein innerer Prozess.

\section{Der Naturzustand UNd DeR GeSellschaftliche Zustand BeI SPINOZA Und Hegel}

Dass das Naturrecht in der Gesellschaft nicht verschwindet und die triebhafte und leidenschaftliche Natur auch im gesellschaftlichen und staatlichen Zustand weiterhin vorhanden bleibt, wird bei Hegel als der «Rest» des Naturzustandes bezeichnet $(\mathrm{R} \$ 19) \cdot{ }^{13}$ Hegel sieht, dass die Menschen leidenschaftlich handeln und dies nicht aufgeben können. Die Reinigung der Triebe ist bei Hegel ein innerer Prozess im Menschen. So zeigt sich, dass auch Hegel den Übergang vom natürlichen zum gesellschaftlichen Zustand im Bewusstseinsgrad des Menschen sieht. Erst durch zwischenmenschliche Beziehungen kann bei Hegel der Mensch zu seinem Selbstbewusstsein gelangen. Der vernünftige Wille kann deshalb erst in der Gesellschaft entstehen. Bei Spinoza entsteht der beständige/ vernünftige Wille ebenfalls in der Gesellschaft, weil er sich erst durch die Gemeinbegriffe bilden kann, die wiederum erst in einer Gesellschaft entstehen können. Ohnehin ist der Mensch immer schon in einem gesellschaftlichen Zustand, weil die Menschen sich gegenseitig brauchen, um überleben zu können. So ist der Übergang vom natürlichen Zustand zum gesellschaftlichen ein Übergang von den Begierden und Leidenschaften zum vernünftigen Willen.

Spinoza behandelt das Naturrecht im zweiten Kapitel des Politischen Traktats. Im Politischen Traktat betont Spinoza, dass das jedem zukommende Recht der Natur im staatlichen Zustand nicht verschwindet. ${ }^{14}$ Hegel spricht in der Einleitung der Grundlinien, von der philosophischen Rechtswissenschaft und sieht die Rechtswissenschaft als ein Teil der Philosophie (R $\$ 2) .{ }^{15}$ Hegel meint, das Naturrecht oder philosophische Recht und Positive Recht verschieden zu sehen ein großes Mißverständnis ist $(\mathrm{R} \S 3) .{ }^{16}$

Spinoza und Hegel haben, was das Verhältnis von Naturzustand und gesellschaftlichem Zustand betrifft, eine andere Auffassung entwickelt als Philosophen wie Hobbes, Rousseau oder Locke und die beiden Zustände nicht als einander entgegengesetzt betrachtet. Dies hängt mit dem «Natur»-Begriff beider

[13] Hegel, GWF 1986, Grundlinien der Philosophie des Rechts, Suhrkamp Verlag, Frankfurt am Main, p. 354.

[14] Spinoza, B 1994, Politischer Traktat, Felix-Meiner Verlag, Hamburg, p. 37.

[15] Hegel, GWF 1986, Grundlinien der Philosophie des Rechts, Suhrkamp Verlag, Frankfurt am Main, p. 30.

[16] Hegel, GWF 1986, Grundlinien der Philosophie des Rechts, Suhrkamp Verlag, Frankfurt am Main, p. 35. 
Philosophen zusammen. ${ }^{17}$ Spinozas und Hegels Auffassung der menschlichen Natur beeinflusst auch die Auffassung bezüglich der beiden Zustände. Die Beziehung zwischen dem Naturzustand und dem gesellschaftlichen Zustandes ist eng mit der ersten und zweiten Natur des Menschen verbunden. Wenn wir den vernünftigen Willen als entwickelte Form des Triebes sehen, können wir den Naturzustand und den gesellschaftlichen Zustand nicht als entgegengesetzt betrachten. Die beiden Zustände bilden keinen Gegensatz, sondern eine Kontinuität von dem einen Zustand zum anderen. Eine solche Interpretation der beiden Zustände kann daher nicht mit der klassischen Auffassung der Vertragstheorie zusammen bestehen. Spinoza sieht den Hauptunterschied darin, dass im staatlichen Zustand alle dasselbe fürchten, dass es für alle ein und denselben Urheber der Sicherheit gibt und damit auch eine und dieselbe Lebensweise, welche die Urteilsfähigkeit nicht aufgibt. ${ }^{18}$ Die Vertragstheorie, die bei Spinoza und Hegel anders als bei Hobbes oder Rousseau interpretiert wird, bringt auch mit sich, dass der allgemeine Wille anders interpretiert wird. Wie entsteht aber bei Spinoza und Hegel der gesellschaftliche Konsens, wenn nicht durch den Vertrag? Der Vertrag kann nach Spinoza und auch Hegel die Dynamik und die sich ständig ändernden Verhältnisse im Staat nicht beisammen halten. Deshalb finden wir den Konsens bei Spinoza und Hegel in den Gesetzen und in der Verfassung. Der Übergang von Naturzustand zum gesellschaftlichen Zustand ist sowohl bei Spinoza als auch bei Hegel nicht ein Übergang von einem Zustand des Krieges untereinander zu einem vernünftigen Zustand, sondern auch in der Gesellschaft hört das Natürliche nicht auf zu existieren. Die Vertragstheorie wird von beiden auf verschiedene Weisen verworfen, und eine natürliche Kontinuität der Gesellschaft wird anstelle des Vertrages gesetzt. Diese Kontinuität hängt vom Bildungsgrad ab. Und die Bildung wird wiederum eng mit den Gesetzen in Verbindung gebracht. ${ }^{19}$ Nach Spinoza entsteht im gesellschaftlichen Zustand durch Sitten, Sprache und Gesetze eine ähnliche Lebensweise. ${ }^{20}$ Die Menschen, die sich durch Furcht oder Hoffnung vereinen, entwickeln durch die Hoffnung oder durch die Furcht die Motive der Lebensweise sozusagen eine zweite Natur. Die zweite Natur wird durch die Verinnerlichung der Werte und Gesetze einer Gesellschaft geprägt. Die zweite Natur ist die entwickelte Form der ersten triebhaften Natur durch

[17] Ilting hat die Beziehung zwischen Spinozas Naturrechtslehre und derjenigen Hegels dargestellt. Seiner Meinung nach sind die Gedanken Hegels eine Weiterführung der Naturrechtlehre Spinozas. Siehe Ilting, K 1974, 'Hegels Auseinandersetzung mit der aristotelischen Politik', G.W.F Hegel, Frühe Politische Systeme, Ullstein Verlag, Frankfurt-Berlin-Wien, pp. 759-786.

[18] Spinoza, B 1994, Politischer Traktat, Felix-Meiner Verlag, Hamburg, p. 37.

[19] Siehe GüngÖRen Bulgan, B 2014, 'Die Beziehung zwischen Bildung und Verfassung bei Hegel', Hegel-Jahrbuch, pp. 214-219.

[20] SpInozA, B 1994, Theologisch-politischer Traktat, Felix-Meiner Verlag, Hamburg, p. 270. 
die Kultur der Gesellschaft. Die zweite Natur ist aber nicht etwas, was die erste Natur auflöst, sondern sie existieren miteinander. ${ }^{21}$ So wird auch das «wie von einem Geist geleitet Sein» (una veluti mente ducitur) ein viel diskutierter Begriff $^{22}$ von Spinoza durch Furcht und Hoffnung bestimmt und nicht durch eine Homogenität.

Auch bei Hegel ist es eine durch die Sittlichkeit heranwachsende zweite Natur, die durch Gewohnheit geprägt ist $(\mathrm{R} \ 4){ }^{23}$ Die «zweite Natur» wird somit an die Stelle der ersten Natur, die als natürlicher Wille verstanden wird, gesetzt. ${ }^{24}$ Die erste Natur entwickelt sich durch Bildung und Arbeit zu einer zweiten Natur (E \$ 410). ${ }^{25}$ Der Volksgeist entwickelt sich auch bei Hegel nicht durch eine homogene Menge, wie sie meist verstanden wird, sondern er ist die Organisation der Menge, die nicht atomisiert sein darf, wenn es um Produktives in der Gesellschaft gehen soll (E $₫ 544) .{ }^{26}$ Die Menge kann ihr Ziel nur

[21] Im TTP wird die Liebe der Hebräer zu ihrem Vaterland, als «zweite Natur» bezeichnet. Diese Liebe war nach Spinoza keine einfache Liebe, sondern eine Frömmigkeit, die ihnen zur «zweiten Natur» wurde. Der tägliche Kult ist von dem der anderen Völker verschieden gewesen, und dadurch unterschieden sie sich von diesem. (SPInOzA, B 1994, Theologisch-politischer Traktat, Felix-Meiner Verlag, Hamburg, p. 267.)

[22] Matheron vertritt die These, dass das «als ob von einem Geist geleitet sein» auf eine organische Theorie hinweist. (Matheron, M 1988, Individu et communauté chez Spinoza, Editions de Minuit, Paris, pp. 347-348.) Laut Rice würde eine organische Theorie Spinoza zu einem Vorläufer von Hegel und Marx machen. Rice versteht diesen Begriff metaphorisch. McShea und er vertreten die These, dass die Bildung der Kollektivitäten keine Individuation, sondern eine Harmonie bilden (Balibar, E 2001, 'Potentia Multitudinis, quae una veluti ducitur', Ethik, Recht und Politik bei Spinoza, Schultess, Zürich, 2001, p. 115).

[23] "(...) das Rechtssystem das Reich der verwirklichten Freiheit, die Welt des Geistes aus ihm selbst hervorgebracht, als seine zweite Natur" (Hegel, GWF 1986, Grundlinien der Philosophie des Rechts, Suhrkamp Verlag, Frankfurt am Main, p. 46).

[24] İbid., p. 301.

[25] HegeL, GWF 2003, Enzyklopädie der philosophischen Wissenschaften im Grundrisse III, Suhrkamp Verlag, Frankfurt am Main, p. 184.

[26] «Analog zum lateinischen Unterschied zwischen «vulgus» und «populus» unterscheidet Hegel zwei Bedeutungen. Einerseits wird das Volk als eine teils natürliche, teils kulturelle Gemeinschaft eingeführt, andererseits wird es als die Vielen, oder als Menge einzelner Individuen, dem Monarchen oder der Regierung entgegengesetzt» (...) Hegel, der anfangs den Begriff «Volk» öfter benutzt, betont in seinen späteren Schriften, dass das Volk nur als Staat imstande ist, nach außen Souveränität und Selbständigkeit zu behaupten (CrusYberghs, P 2006, 'Volk', Hegel-Lexikon, Ed. Paul Cobben, Paul Cruysberghs, Peter Jonkers, Wissenschaftliche Buchgesellschaft, p. 215). Das Aggregat der Privaten pflegt häufig das Volk genannt zu werden; als solches Aggregat ist es aber vulgus, nicht populus; und in dieser Beziehung ist es der alleinige Zweck des Staates, dass ein Volk nicht als solches Aggregat zur Existenz, zur Gewalt und Handlung komme. (...) Das Volk wäre in einem solchen Zustand eine unförmliche, wüste, blinde Gewalt, wie die des aufgeregten Meeres (Hegel, GWF 2003, Enzyklopädie der philosophischen Wissenschaften 
erreichen, wenn sie sich organisiert, und nur so kann sie sich eine Verfassung geben. Und dies zeigt die Beziehung des Geistes eines Volkes zur Verfassung.

\section{Die Institutionen-Logik Bei Spinoza und Hegel}

Spinoza und Hegel versuchen die Institutionen im Staat zu verwirklichen, indem sie in ihren Entwürfen die affektive und leidenschaftliche Natur der Regierenden, die ein willkürliches Handeln verursacht, in Auge zu behalten und durch die Gesetze und die Teilung der Macht zu verhindern suchen. Doch auch die der Bürger werden im Staat, um willkürliches Handeln zu verhindern, durch subjektive Rechte eingegrenzt. Gleichzeitig wird aber erst gerade durch diese subjektiven Rechte ein Handlungsraum für die Individuen möglich. Spinozas Selbstbewegung der Menschen zur Freiheit ist auch als «List der Affekte» oder «List der Vernunft» charakterisierbar. ${ }^{27}$ Seine Theorie, die durch subjektive Unvernunft hindurch ein vernünftiges Institutionensystem herstellt, nimmt die Hegelsche Figur der «List der Vernunft» vorweg. ${ }^{28}$ Um den Vergleich der Institutionen zu konkretisieren kann man bei beiden Denkern das Beispiel der Monarchie herausarbeiten. Spinoza versucht in verschiedenen Regierungsformen (Aristokratie, Monarchie und Demokratie), eine Balance zwischen Regierenden und Regierten herauszuarbeiten. Dagegen konzentriert sich Hegel eher auf die Monarchie.

Hegel sieht wie Spinoza in den Institutionen die entlastende Funktion ${ }^{29}$ für das Individuum. Spinoza und Hegel betrachten beide den Menschen mit seinen Interessen in der Gesellschaft und sehen, dass die Menschen für ihr eigenes Interesse handeln. Die Frage ist, wie die Interessen in gegenseitiger Befriedigung organisiert werden können. Beide sehen in den staatlichen Institutionen einen Mechanismus, der die Kluft zwischen bewusstem und unbewusstem Handeln durch die List der Affekte/Vernunft überwinden und die vernünftigen Ordnung gewährleisten kann. Spinoza und Hegel versuchen, das Recht objektiv ohne Abhängigkeit von subjektiven Entscheidungen der Einzelnen zu denken. ${ }^{30}$ Es

im Grundrisse III, Suhrkamp Verlag, Frankfurt am Main, p. 341).

[27] Walther, M 1990, 'Politik, Moralität und Freiheit in der Philosophie Spinozas. Die Destruktion der «Praktischen» Philosophie und die Begründung einer «analytischen» Wissenschaft von der Politik in der Neuzeit', Selbstbehauptung und Anerkennung, Ed. H GIRndT, Academia Verlag, p. 20.

[28] Walther, M 2007, 'Souveränität, Ausnahmezustand und Widerstandsrecht bei Spinoza', Spinoza: Individuo e Moltitudine, Società Editrice «Il Ponte Vecchio», p. 400.

[29] Siehe Walther, M 1990, 'Institution, Imagination und Freiheit bei Spinoza', Politische Institutionen im Gesellschaftlichen Umbruch, Westdeutscher Verlag, Opladen, pp. 246-275.

[30] Macherey schreibt, dass Spinoza und Hegel ein subjektloses Recht denken in dem Sinne, dass das Recht nicht von den Einzelnen abhängig ist (Macherey, M 1992, Avec Spinoza. Etudes sur la doctrine et l'histoire du spinozisme, PUF, Paris, p. 140). 
sind nicht nur diejenigen, die regiert werden, sondern auch die Regierenden, die durch die Institutionen zum vernunftgemäßen Handeln gebracht werden. Spinoza konkretisiert diese Situation durch das Beispiel des Odysseus. ${ }^{31}$ Wenn wir auch bei Hegel von dem Beispiel in der Monarchie ausgehen, können wir sagen, dass der Monarch, das Pünktchen auf das I setzt. ${ }^{32}$ Nach Hegel wird in einer gebildeten Verfassung durch die vernünftige und feste Organisation des Staates die Individualität des Monarchen unwichtig, und die Kraft der Vernünftigkeit dieser Verfassung liegt gerade in dieser Unbedeutsamkeit. ${ }^{33}$ Der Ausdruck «Festigkeit gegen die Leidenschaft» zeigt, dass auch Hegel der Meinung ist, dass der Monarch gar nicht dahin gebracht werden soll, willkürlich zu handeln, sondern seine Minister um Rat fragen muss und so für die Regierungshandlungen völlig unverantwortlich ist. ${ }^{34}$ Neben der entlastenden Funktion haben die Institutionen auch eine befreiende Funktion. Diese kann nur real werden, wenn die Institutionen sich den gesellschaftlichen Änderungen anpassen können. Sie können so unter gewissen Umständen die Selbstverwirklichung des Einzelnen ermöglichen.

Die Institutionen, die bei Spinoza und Hegel eine entlastende Funktion haben, veranlassen die Menschen, obwohl sie affektiv handeln, bei der Verfolgung ihrer eigenen Interessen doch vernunftgemäß zu handeln. Denn durch den gegenseitigen Nutzen entstehen Erwartungen, die durch Institutionen reguliert werden. Der Mechanismus im Staat muss so beschaffen sein, dass er unabhängig von den Einzelnen funktioniert. Wichtig ist es, eine Zirkularität zwischen dem

[31] «Die Gefährten des Odysseus führten nämlich seine Anordnungen aus, als sie sich weigerten, ihn, der am Schiffsmast angebunden und vom Sirenengesang bezaubert war, loszubinden, obwohl er es ihnen unter vielfachen Drohungen befahl, und es wird ihm als Weisheit angerechnet, dass er später seinen Gefährten dafür, dass sie seiner ersten Willensäußerung gehorcht hatten, gedankt hat. Diesem Beispiel des Odysseus folgend, pflegen sogar Könige die Richter anzuweisen, Gerechtigkeit auszuüben, ohne auf jemanden besondere Rücksicht zu nehmen, selbst nicht auf den König, wenn er in einem bestimmten Fall etwas anordnet, von dem sie wissen, dass es dem geltenden Recht widerstreitet» (SpINOzA, B 1994, Politischer Traktat, Felix-Meiner Verlag, Hamburg, pp. 93-97).

[32] «Das ,Ich will' muss als Person gefasst werden, was aber nicht heißt, dass der Monarch willkürlich handeln kann» (HegeL, GWF 1986, Grundlinien der Philosophie des Rechts, Suhrkamp Verlag, Frankfurt am Main, p. 449). «Denn die Spitze (des Staates) soll so sein, dass die Besonderheit des Charakters nicht das Bedeutende ist» (Hegel, GWF 1986, Grundlinien der Philosophie des Rechts, Suhrkamp Verlag, Frankfurt am Main, p. 451).

[33] Petersen ist der Meinung, dass Hegels Monarch kaum mehr vermag als der Präsident der Bundesrepublik Deutschland (Petersen, T 2002, 'Republikanismus und politische Tugend bei Hegel', Staat, Recht und Politik beim frühen Hegel, Ed. M Henkel, Berliner Wissenschaftsverlag, Berlin, p. 155).

[34] Hegel, GWF 1983, Philosophie des Rechts, Die Vorlesung von 1819/20 in einer Nachschrift, Ed. D. Henrich, Suhrkamp Verlag, Frankfurt am Main, p. 253. 
Volk/Menge und den Regierenden herzustellen. Die Subjektivität sowohl der Regierenden als auch der Regierten ist begrenzt, und doch muss auch sie in einem Rahmen, der den anderen nicht schadet, ausgeübt werden können. Denn sie kann nicht zu lange unterdrückt werden, weil der Mensch - wie Spinoza meint - es von Natur aus nicht aushält, nicht seine Meinung zu sagen ${ }^{35}$, oder weil der Mensch - wie Hegel sagt - den prickelnden Trieb hat, seine Meinung zu sagen ( $\mathrm{R} \$ 319) .{ }^{36}$ Es muss dem Individuum im Staat ein großmöglichster Freiraum geschaffen werden, damit es sich realisieren kann, weil es sonst für den Staat und die Gesellschaft zerstörerisch wird. Dieses Moment der Meinungsfreiheit ist daher nicht nur für das Individuum wichtig, sondern auch für die gesamte Gesellschaft. Sie ist - wie Hegel es ausdrückt - für die Bildung des Einzelnen unverzichtbar, weil in der öffentlichen Meinung verschiedene Meinungen aufeinander treffen und «eine Gescheitheit eine andere auffrisst». In Hegels Grundlinien ist sie das unorganische Moment, aber gleichzeitig das Moment, in dem sich aus dem Unorganischen eine Organisation bildet. Jedoch hat die Meinungsfreiheit im Staat bei Spinoza auch bei Hegel ihre Grenzen. Ein weiteres unorganisches Moment im Staat ist die Empörung oder der Widerstand. Dies ist die extreme Ausformung der Subjektivität. Doch auch eine Empörung kann organisiert werden, und sie entwickelt sich zu einem Aufstand oder zu einer Revolution. Ein gesetzlich anerkanntes Widerstandsrecht im Staat gibt es allerdings weder bei Hegel noch bei Spinoza. Bei Spinoza ist es jedoch mit dem Naturrecht, das der Mensch niemals abgibt, verbunden, und Hegel betrachtet es als ein historisches Faktum.

\section{Der Macht-Begriff und der Aufbau Der ZwischenstaAtlichen BeZiehungen}

Die Zwischenstaatlichen Beziehungen können bei Spinoza und Hegel analog zu den Individuen gedacht werden. Der Naturzustand der Staaten ist der Krieg, so wie es bei den Individuen die Triebe und Leidenschaften sind. Selbstbestimmtheit, in Bezug auf die Staaten Souveränität genannt, ist wie die Selbstbestimmtheit der Individuen ohne Kontakt zu anderen Individuen/ Staaten eine Selbsttäuschung. Die Staaten müssen erst Kontakte zu anderen Staaten aufnehmen, Bündnisse schließen, um ihre Souveränität behaupten zu

[35] Seine Ideen über die Meinungsfreiheit entwickelt Spinoza im 20. Kapitel des Theologisch Politischen Traktats. Es hei $\beta \mathrm{t}$, dass niemand «sein natürliches Recht oder seine Fähigkeit, frei zu schließen und über alles zu urteilen, auf einen anderen übertragen noch kann er zu einer solchen Übertragung gezwungen werden» (SpINozA, B 1994, Theologisch-politischer Traktat, Felix-Meiner Verlag, Hamburg, p. 299).

[36] Hegel, GWF 1986, Grundlinien der Philosophie des Rechts, Suhrkamp Verlag, Frankfurt am Main, pp. 486-489. 
können. Durch Anpassung entsteht wie bei den Individuen eine überstaatliche Vernunft, die durch Institutionen entwickelt wird. Der vernünftige Wille in Bezug auf die Staaten, Frieden genannt, kann sich also nur durch Kooperation entwickeln. Bei der Nützlichkeit kann man auch eine Analogie zu den zwischenmenschlichen Beziehungen herstellen. Internationale Verträge werden nach der Auffassung von Spinoza und Hegel nur so lange anerkannt, wie sie als nützlich angesehen werden.

Das «clausula rebus sic stantibus»-Prinzip ist sowohl bei Spinoza als auch bei Hegel das Geltungs-Prinzip zwischenstaatlicher Verträge. Die Ablehnung des Pacta sunt servanda-Prinzips macht Spinoza zu einem Vorläufer von Hegel. ${ }^{37}$ Die Staaten wie die Individuen folgen bei Spinoza ihrem Nutzen und halten sich an die Verträge, solange die Verträge für sie einen Profit bringen. Ein ähnliches Argument können wir auch bei Hegel wiederfinden. Die Verträge zwischen den Staaten beruhen nach Hegel auf den besonderen Willen der Staaten und sind deshalb relativ und beschränkt ( $\mathrm{R} \S 336)$. Das heißt, sie sind relativ, weil zu jeder Zeit ein Staat den Vertrag brechen kann, wenn dieser für ihn nicht mehr nützlich ist. Der Übergang der Staaten zu einer völkerrechtlichen Ordnung ist nach Hegel nicht durch Verträge möglich, weil diese, wenn sie für die Staaten keinen Vorteil mehr bringen, gleich verworfen werden können.

Ein zweiter Punkt, in dem Hegel bezüglich der zwischenstaatlichen Beziehungen mit Spinoza zusammen erwähnt wird, ist der Macht-Begriff ${ }^{38}$ und, mit diesem Begriff verbunden, die Naturrechtsauffassung. ${ }^{39}$ Die Machtbeziehung

[37] Wehberg, H 1959, 'Pacta Sunt Servanda', American Journal of International Law, Vol. 53, p. 779.

[38] Der Begriff der Macht hat bei Spinoza nicht einen ausschließlich physischen Charakter. Menschen üben gerade Kraft ihrer Sprache und ihres Denkvermögens Macht aus und können diese durch die Reflexion der affektiven Bestimmung optimieren. (Cheneval, F 2001, 'Spinozas Philosophie der internationalen Beziehungen', Ethik, Recht und Politik bei Spinoza, Schultess, Zürich, p. 198). Pažanin macht darauf aufmerksam, dass Hegels Staat nicht nur als Machtstaat, sondern auch als Kulturstaat begriffen werden muss (PAžAnIN, A 1990, 'Sittlichkeit als «zweite Natur» des Menschen', Hegel-Jahrbuch, p. 241).

[39] Nach den Veröffentlichungen der Rechtsphilosophie wurde von einem anonymen Rezensenten auf den Einfluss von Spinozas Naturrecht in Hegels Werk aufmerksam gemacht. Das alte Naturrecht und der Spinozismus fallen diesem Rezensenten nach im Recht des Stärkeren zusammen. So sehen wir, dass der Rezensent mit der Naturrechts-Auffassung das Recht des Stärkeren und somit den Machtstaatsgedanken verbindet (Anonym, Allgem. Lit. - Ztg., 20.-22. 1822 (Nr. 45-47) 353-371, Hegel, GWF 1974, Rechtsphilosophie, Ed. K. Ilting, FrommannHolzboog, Stuttgart-Bad-Cannstatt, p. 467-490). Hegels Prinzip «das Wirkliche ist vernünftig und das Vernünftige ist wirklich» ist, nach Lauterpacht, eine andere Formulierung der Konzeption jus naturale von Spinoza (LauterpaCHT, H 1975, 'Spinoza and international Law', International Law of Peace, Cambridge University Press, Cambridge, p. 381). Die Umwandlung des neuzeitlichen Naturrechts spiegelt bei Hegel die mit Hobbes, Machiavelli und Spinoza verbundene Politik wider 
zwischen den Staaten ist mit der Kriegsverherrlichung in Verbindung gebracht worden, und so sahen viele Spinoza und Hegel als Leugner des Völkerrechts. ${ }^{40}$ Es wurde behauptet, dass Spinoza Hegel präludierte, ${ }^{41}$ und sogar, dass er in den Grundlinien der Philosophie des Rechts Passagen des Tractatus Politicus paraphrasiere, besonders wenn er schreibt, dass die Staaten sich im Naturzustand befinden und dass die Staaten, wenn sie sich nicht einigen können, Kriege führen. ${ }^{42}$ Der Machtstaatsgedanke ${ }^{43}$ sollte aber bei Spinoza und Hegel nicht als eine Kriegsverherrlichung verstanden werden, sondern kann als eine Darstellung der realistischen Verhältnisse gesehen werden. Spinoza sieht den Frieden als eine Tugend und nicht als Abwesenheit des Krieges. Damit könnte man sagen, dass der Friede die «zweite Natur» zwischen den Staaten bildet, und der Krieg den leidenschaftlichen Zustand der Staaten, die «erste Natur»

und führt nach Ottmann vom praktischen Wissen zur pragmatischen Kunst der Machttechnik. (Ottmann, H 1977, Individuum und Gemeinschaft bei Hegel, Berlin/ New York, De Gruyter, p. 311). Ottmann meint, dass Rechtshegelianer und liberale Kritiker ihr Hegel-Bild bestätigt sehen können, wenn trotz der Entzweiungslehre, also der Kritik am modernen individualistischen Naturrecht, an universalistischen Ständelehren und autoritären Regierungsmodellen eine Art von Spinozismus und Kriegsverherrlichung sowie ein Überwiegen der Natur im Begriff der Sittlichkeit dominieren und eigentlich erst in den Jenenser Jahren die Wende zur Geistesphilosophie das Recht der Natur im Geist durch ein Recht des Geistes in der Natur ablöst (Oтtmann, H 1977, Individuum und Gemeinschaft bei Hegel, Berlin/ New York, De Gruyer, p. 311. 391).

[40] Nussbaum, A 1960, Geschichte des Völkerrechts, Beck’sche Verlagsbuchhadlunng, München und Berlin, p. 163.

[41] Meinecke, F 1957, Die Idee der Staatsräson in der neueren Geschichte, R. Oldenburg Verlag, München, p. 272.

[42] Lauterpacht, H 1975, 'Spinoza and international Law', International Law of Peace, Cambridge University Press, Cambridge, pp. 381-382.

[43] Kiesewetter sagt, dass Spinoza die Formel ubi vis, ibi jus prägte und dass sie die machtstaatliche Konzeption ist, die in den Rechtshegelianismus übergegangen ist (KIESEWETTER, H 1995, Von Hegel zu Hitler. Die politische Verwirklichung einer totalitären Machtstaatstheorie in Deutschland (1815-1945), Peter Lang Verlag, Frankfurt am Main, p. 152). Nachdem Heller auf den Einfluss Machiavellis auf Hegel aufmerksam gemacht hat, schreibt er, dass Hegels Staatsauffassung gleich bedeutende, wenn nicht noch stärkere Anregung von Spinoza empfing. Heller betont die Radikalität von Spinozas Lehre von Staat und Macht und meint, dass Spinozas Einfluss auf Hegel grundlegend gewesen sei, weil sich die beiden Denker in ihren monistischen Grundvoraussetzungen sehr nahe standen. Heller sagt, dass Hegel die in der Lehre von Spinoza und Machiavelli empfangenen Anregungen in seiner Staatslehre mit eingebaut hat, von wo aus sich die Tradition der «Machtlehre» bis in unsere Tage verfolgen lässt (Heller, H 1961, Hegel und der Nationale Machtstaatsgedanke in Deutschland, Otto Zeller Verlagsbuchhandlung, Aalen, p. 57). Auch wenn Heller den Machtstaatsgedanken hervorhebt, meint er, dass die Macht des Staates aber nicht als militärisch-politische verstanden werden darf, sondern als eine ethischhistorische (Heller, H 1961, Hegel und der Nationale Machtstaatsgedanke in Deutschland, Otto Zeller Verlagsbuchhandlung, Aalen, p. 88). 
darstellt. Die durch Kooperation immer enger werdenden Beziehungen führen in Spinozas Theorie zu einem zwischenstaatlichen Frieden, weil der Staat entweder aus Furcht vor der Macht der anderen Staaten oder wegen des Nutzens, der durch diese Beziehung entsteht, mehr Rücksicht auf andere Staaten nehmen muss. Durch gegenseitige Anerkennung und Kooperation zwischen immer mehr Staaten wird die Gefahr der Kriege immer geringer. Spinoza betont die immer enger werdenden Beziehungen zwischen den Staaten. Souveränität ohne Anerkennung zu behaupten, hat für Hegel an und für sich keine Bedeutung. Es sind die Gewohnheit und Sitten, die nach Hegel die Bedingungen eines Völkerrechtes ausmachen. Bei Hegel sehen wir die «Anerkennung der Staaten» als ein wichtiges Prinzip, das zu einem Bund der Staaten führen kann ( $\mathrm{R} \$ 33$ ). Nur durch die Sittlichkeit, die sich durch die gegenseitige Abhängigkeit und den Nutzen im zwischenstaatlichen Raum bildet, kann von einem Völkerrecht die Rede sein. ${ }^{44}$ Durch eine immer stärker im zwischenstaatlichen Bereich sich bildende Sittlichkeit kann es zu einem Völkerrecht kommen, das auch Stabilität hat. Hegel beendet die Grundlinien mit der Weltgeschichte. Bei Spinoza sind die Prämissen über das Völkerrecht im dritten Teil gelagert.

\section{FAZIT}

Es wird durch die Analyse mancher Begriffe deutlich, dass Spinoza und Hegel - auch wenn Sie verschiedene Begriffe benutzen und eine grundverschiedene Systematik im Aufbau vieler Begriffe verwenden - einen ähnlichen Weg gehen. Hegel ist von manchen Begriffen von Spinoza beeinflusst, wie z.B. von dem Freiheits-Begriff und auch dem Macht-Begriff. Dieser Einfluss, wenn er auch nicht an vielen Stellen explizit erwähnt wird, zeigt sich in vielen anderen Begriffen auch implizit. In diesem Vortrag wurden die Begriffe analysiert, die einen ähnlichen Aufbau besitzen und doch eine ganz verschiedene Stellung in den Texten von Spinoza und Hegel einnehmen. Es wurde außerdem versucht, die Begriffe von Spinoza und Hegel einander nahe zu bringen um einen Vergleich zu ziehen. Der erste Begriff, der in diesem Zusammenhang analysiert wird, ist der vernünftige oder beständige Wille bei Spinoza und der objektive Wille, der auch als vernünftiger Wille bei Hegel bezeichnet wird. Beide Denker sehen den vernünftigen Willen auf Grund der triebhaften Natur der Menschen als erforderlich an und sehen den vernünftigen Willen nur im Staat verwirklichbar. Die Beziehung der Begriffe Freiheit und Notwendigkeit, welche bei beiden Denkern zusammengedacht werden, beeinflussen auch die Auffassung von Naturzustand und Gesellschaftlichem Zustand, welche nicht als entgegengesetzt, sondern zusammen gedacht werden, weil die Spannung zwischen Vernunft und Unvernunft nicht aufhört. Diese Spannung beeinflusst

[44] Kley, R 1999, Der Frieden unter Demokratien, Stämpfli Verlag, Wien, pp. 17; 24. 
auch die Institutionen-Logik beider Denker. Die List der Vernunft ist ein Begriff, der diesbezüglich bei beiden Denkern auftritt. Ein anderer wichtiger Begriff, von dem Hegel von Spinoza beeinflusst worden ist, ist der Macht-Begriff. Doch in den Ausführungen über die zwischenstaatlichen Beziehungen sind noch andere Parallelen zu finden. Der gegenseitige Nutzen und die Abhängigkeit der Staaten sind bei Spinoza kennzeichnend und werden bei Hegel durch die Anerkennungs-Logik deutlich. Es wird offenbar, dass neben Begriffen Spinozas, die von Hegel übernommen worden sind, bei einer weiteren, tieferen Analyse der Architektonik und Struktur mancher Begriffe und Gedankengänge noch weitere Parallelen entdeckt werden können.

\section{BIBLIOGRAPHIE}

Balibar, E 2001, 'Potentia Multitudinis, quae una veluti ducitur', Ethik, Recht und Politik bei Spinoza, Schultess, Zürich, 2001, pp. 105-137.

Cheneval, F 2001, 'Spinozas Philosophie der internationalen Beziehungen', Ethik, Recht und Politik bei Spinoza, Schultess, Zürich, pp. 195-205.

Crusyberghs, P 2006, 'Volk', Hegel-Lexikon, Ed. Paul Cobben, Paul Cruysberghs, Peter Jonkers, Wissenschaftliche Buchgesellschaft.

Güngören Bulgan, B, Die Bedeutung von Hegels Spinoza-Lektüre für seine Staatsund Rechtstheorie, Logos, Berlin, 2011.

Güngören Bulgan, B 2014, 'Die Beziehung zwischen Bildung und Verfassung bei Hegel', Hegel-Jahrbuch, pp. 214-219.

Hegel, GWF 1983, Philosophie des Rechts, Die Vorlesung von 1819/20 in einer Nachschrift, Ed. D. Henrich, Suhrkamp Verlag, Frankfurt am Main

Hegel, GWF 1986, Grundlinien der Philosophie des Rechts, Suhrkamp Verlag, Frankfurt am Main.

Hegel, GWF 2003, Enzyklopädie der philosophischen Wissenschaften im Grundrisse, Suhrkamp Verlag, Frankfurt am Main.

Hegel, GWF 2003, Vorlesungen über die Geschichte der Philosophie III, Suhrkamp Verlag, Frankfurt am Main.

HegeL, GWF 1974, Rechtsphilosophie, Ed. K. Ilting, Frommann-Holzboog, StuttgartBad-Cannstatt.

Heller, H 1961, Hegel und der Nationale Machtstaatsgedanke in Deutschland, Otto Zeller Verlagsbuchhandlung, Aalen.

IttinG, K 1974, 'Hegels Auseinandersetzung mit der aristotelischen Politik', G.W.F. 
Hegel, Frühe Politische Systeme, Ullstein Verlag, Frankfurt-Berlin-Wien, pp. 759-786.

Kiesewetter, H 1995, Von Hegel zu Hitler. Die politische Verwirklichung einer totalitären Machtstaatstheorie in Deutschland (1815-1945), Peter Lang Verlag, Frankfurt am Main.

KLEY, R 1999, Der Frieden unter Demokratien, Stämpfli Verlag, Wien.

LaUterpacht, H 1975, 'Spinoza and international Law', International Law of Peace, Cambridge University Press, Cambridge, pp. 366-384.

Lucas, H 1982-83, 'Hegel et l'édition de Spinoza par Paulus (1)', Cahiers Spinoza, Numéro 4, Editions Réplique, pp. 127-138.

Lucas, H 1999, 'Der junge Hegel zwischen Revolution und Reform. Politische und rechtsphilosophische Optionen Hegels Übergang von Bern nach Frankfurt', Hegels Denkentwicklung in der Berner und Frankfurter Zeit, Ed. M. Bondeli; H. Linneweber-Lammerskitten, Fink Verlag, München, pp. 251-276.

Macherey, A 1992, Avec Spinoza, Etudes sur la doctrine de l'histoire du spinozisme, Puf, Paris.

Matheron, M 1988, Individu et communauté chez Spinoza, Editions de Minuit, Paris.

Meinecke, F 1957, Die Idee der Staatsräson in der neueren Geschichte, R. Oldenburg Verlag, München.

Nussbaum, A 1960, Geschichte des Völkerrechts, Beck'sche Verlagsbuchhadlunng, München und Berlin.

Ottmann, H 1977, Individuum und Gemeinschaft bei Hegel, Berlin/ New York, De Gruyer.

PAŽAnin, A 1990, 'Sittlichkeit als «zweite Natur» des Menschen', Hegel-Jahrbuch, pp. 235-242.

Petersen, T 2002, 'Republikanismus und politische Tugend bei Hegel', Staat, Recht und Politik beim frühen Hegel, Ed. M Henkel, Berliner Wissenschaftsverlag, Berlin.

Rотн, K 1991, Die Institutionalisierung der Freiheit in den Jenaer Schriften Hegels, Schäuble Verlag, Rheinfelden.

SpinozA, B 1994, Politischer Traktat, Felix-Meiner Verlag, Hamburg.

SpInozA, B 1994, Theologisch-politischer Traktat, Felix-Meiner Verlag, Hamburg.

Walther, M 1990, 'Institution, Imagination und Freiheit bei Spinoza', Politische Institutionen im Gesellschaftlichen Umbruch, Westdeutscher Verlag, Opladen, pp. 246-275.

Walther, M 1990, 'Politik, Moralität und Freiheit in der Philosophie Spinozas. Die Destruktion der «Praktischen Philosphie und die Begründung einer 
«analytischen» Wissenschaft von der Politik in der Neuzeit', Selbstbehauptung und Anerkennung, Ed. H Girndt, Academia Verlag, pp. 7-23.

Walther, M 2007, 'Souveränität, Ausnahmezustand und Widerstandsrecht bei Spinoza', Spinoza: Individuo e Moltitudine, Società Editrice «Il Ponte Vecchio», p. 391-400.

Wehberg, H 1959, 'Pacta Sunt Servanda', American Journal of International Law, Vol. 53, pp. 775-786.

BIRDEN GUNGÖREN is assistant professor at the Galatasaray University Faculty of Law

RESEARCH INTERESTS:

Theory of State and Law, Theory of Human Rights

AdDress:

Galatasaray Üniversitesi Hukuk Fakültesi, Çırağan caddesi no:36, 34349

Ortaköy, İstanbul, Türkiye

\section{ReCEnt Publications:}

- Die Bedeutung von Hegels Spinoza-Lektüre für seine Staats- und Rechtstheorie, Logos Verlag, Berlin, 2011

- «Wandel des Strafrechts am Beispiel des Deutschen Idealismus» Yeditepe Üniversitesi Hukuk Fakültesi Dergisi, Duygun Yarsuvat'a Armağan, Cilt 9, Say12, Y1l 2012, s.523-549.

- «Die Beziehung zwischen Bildung und Verfassung bei Hegel», Hegel-Jahrbuch, 2014, s.214-220.

-«Faydacı ve Tarihçi Hukuk Okulunun İnsan Hakları Eleştirileri», ( The Human Rights Critique of The Utilitarian and Historical Law School), Galatasaray Üniversitesi Hukuk Fakültesi Dergisi, Melike Batur Yamaner'in Anısına Armağan, Cilt 1/2014, 261-303

- «Critics of Human Rights from a historical Perspective», Human Rights, Rule of Law and the Contemporary social Challenges in Complex Societies: Proceedings of the XXVI World Congress of Philosophy of Law and Social Philosophy, edited by Marcelo Galuppo, Mônica Sette Lopes, Lucas Gontijo, Karine SalGado, Thomas Bustamante., 1st ed., Belo Horizonte: Initia Via, 2015, http:// www.initiavia.com/ivr-2013 s.2383-2396 